Steel, R. \& Walker, T. K. (1957). J. gen. Microbiol. 17, 12-18

\title{
Celluloseless Mutants of certain Acetobacter Species
}

\author{
By R. STEEL AND T. K. WALKER \\ Manchester College of Science and Technology
}

SUMMARY: The occurrence has been studied of mutants which did not produce extracellular cellulose in cultures of two strains of Acetobacter acetigenum (NCIB 8132, NCIB 5346) and one strain of $A$. xylinum var. africanum (NCIB7029). With about 150 glucose yeast-extract (GYE) medium static cultures of organism NCIB8132, prepared over a period of about one year, cellulose pellicles were produced in each case. In contrast, the loss of cellulose-forming ability and the appearance of diffuse general growth were noted with 6 out of 26 tests made with static cultures in an ethanol yeast-extract (EYE) medium, 3 out of 905 isolates selected from platings of GYE shaken cultures, and 1 out of 3 tests made with EYE shaken cultures. By a 'mass-plating' technique it was found that mutants which did not produce cellulose pellicles ('celluloseless mutants') were present in cellulose-producing cultures. The two other cultures (NCIB 5346, NCIB 7029) underwent mutation spontaneously in GYE static cultures on several occasions.

The present work was undertaken to obtain mutants of Acetobacter acetigenum and $A$. xylinum var. africanum which did not form extracellular cellulose as did the parent organisms; these mutants will be referred to as 'celluloseless'. Schramm \& Hestrin (1954) reported the occurrence of celluloseless mutants of $\boldsymbol{A}$. xylinum; the mutants appeared when the wild-type organism was grown by serial transfer in 'swirl' cultures on a medium containing glucose, yeast-extract, peptone and salts. Creedy, Jowett \& Walker (1954) isolated a celluloseless mutant of $A$. acetigenum when the parent organism was cultivated in the presence of sodium arsenite $(0.0005 \mathrm{M})$. Further, Wright \& Walker (1955) found that isolates incapable of forming cellulose were present when $A$. acetigenum was grown under agitated and aerated conditions. The present paper deals primarily with the occurrence of celluloseless mutants when the wild-type of $\boldsymbol{A}$. acetigenum was grown under various cultural conditions.

\section{METHODS}

Organisms. Two strains of Acetobacter acetigenum (NCIB 8132, NCIB 5346) and one of $A$. xylinum var. africanum (NCIB 7029) were obtained from the National Collection of Industrial Bacteria, (NCIB), Teddington, Middlesex. All cultures were replated and re-isolated twice and examined microscopically for purity before use.

Media. Glucose yeast-extract (GYE) medium ( $\mathrm{pH} 7 \cdot 0$ ) contained $3 \%$ $(\mathrm{w} / \mathrm{v})$ glucose and $0.5 \%(\mathrm{w} / \mathrm{v})$ Difco yeast extract; for solid media $2 \%(\mathrm{w} / \mathrm{v})$ of agar was added. Ethanol yeast-extract (EYE) medium contained $2 \%$ $(\mathrm{v} / \mathrm{v})$ ethanol and $0.5 \%$ yeast extract, the sterile ethanol being added aseptically after sterilization of the basal medium. Tests were also made with ethylene glycol or $n$-propanol (both at $2 \%, \mathrm{v} / \mathrm{v}$ ) substituted for ethanol. 
Peptone medium consisted of a $5 \%(w / v)$ aqueous solution of Evans's peptone. Malt wort medium ( $\mathrm{pH} \mathrm{5.5)}$ was prepared by the method of Tosic \& Walker (1946). In the early stages of this work the stock cultures of the parent organisms were kept on GYE medium. However, as indicated later, malt wort was found to be better for this purpose and accordingly was used thereafter. Cultures were incubated at $30^{\circ}$ except when otherwise stated.

Static cultures were made either with $30 \mathrm{ml}$. of medium in $100 \mathrm{ml}$. conical flasks or with $8 \mathrm{ml}$. of medium in test tubes $(18 \times 155 \mathrm{~mm}$.).

Shaken cultures. The transfer procedure of Schramm \& Hestrin (1954) was followed. Duplicate conical flasks $(1500 \mathrm{ml}$.) which contained $100 \mathrm{ml}$. of the desired medium were inoculated from a $48 \mathrm{hr}$. GYE test-tube culture by dipping the cellulose pellicle in the medium. The flasks were placed on a reciprocating shaker (55 cycles/min.; $6.5 \mathrm{~cm}$. stroke) in a room controlled at $28 \pm 1^{\circ}$. At 3-day intervals transfers were made with a sterile wire loop from each of the duplicates to two sterile flasks of fresh medium, and one flask of each pair was incubated statically while the other was replaced on the shaker. According to Schramm \& Hestrin (1954) the appearance of turbidity (due to diffuse growth of organisms) in the static cultures indicates the presence of celluloseless mutants. When turbidity was observed, the shaken cultures were plated on GYE agar medium and isolated colonies were picked to GYE liquid medium to observe for cellulose pellicle formation.

Plating on a selective nutrient medium for mutants. A medium was prepared as follows: $20 \mathrm{~g}$. yeast extract (Difco) were shaken with $200 \mathrm{ml}$. of a mixture of ethanol and water $(4: 1)$ in a separating funnel; the supernatant liquid was decanted and the ethanol removed from it under vacuum (below $40^{\circ}$ ). Then the residue was made up to a final volume of $40 \mathrm{ml}$. with water. This solution (1 ml.) + glucose (3 g.) + agar (2 g.), made up to $100 \mathrm{ml}$. with water, comprised the selective medium ( $\mathrm{pH} 6 \cdot 2)$.

The organisms used for plating were obtained from $48 \mathrm{hr}$. GYE parent cultures, each grown from a different single-colony isolate. Inoculum suspensions were prepared by removing the pellicles and washing them in several changes of sterile water, after which they were finally shaken in $5 \mathrm{ml}$. of water to remove as many organisms as possible from the pellicle. Volumes $(c .1 \mathrm{ml}$.) of this last suspension were added to each Petri dish and mixed with $15 \mathrm{ml}$. of selective medium; six replicate plates were prepared with each isolate. Incubation was for 12 days.

Replica plating on the selective medium. The method of Lederberg \& Lederberg (1952) was followed.

Mass-plating on GYE medium. This method for the detection of mutants in the parent culture arose from the observations (made during the present study) that when a sufficiently large inoculum from a cellulose-producing culture was spread on the surface of GYE agar, growth of mutant clones could be observed. Mutant colonies have a faster growth rate than parent colonies and also their colony type is different from that of wild-type organisms.

Each culture (in GYE medium) grown from a single-colony isolate, was well shaken to dislodge as many organisms as possible from the cellulose 
pellicle. Then the desired volume $(0.10$ or $0.20 \mathrm{ml}$.) of inoculum, removed aseptically, was spread with a sterile glass rod over the surface of GYE agar and incubated.

\section{RESULTS}

Experiments with Acetobacter acetigenum NCIB 8132

Static cultures in GYE medium. Over a period of about one year more than 150 cultures were prepared; in no case was the loss of ability to produce cellulose observed.

Static cultures in EYE medium. In 10 experiments which included work with 26 cultures, the following results were obtained: 12 cultures produced cellulose pellicles, 8 gave no visible growth, and 6 produced diffuse growth with no visible cellulose pellicle. The cultures which formed cellulose pellicles grew very slowly in this medium; the pellicles appeared after 3-5 days of incubation. The appearance of diffuse growth generally occurred, if at all, between 24 and $48 \mathrm{hr}$. of incubation, although in some cases it appeared as late as 5 days. It is apparent from these results that the parent culture did not grow normally in EYE medium, since cellulose pellicles appeared in only 12 of the 26 tests; the production of cellulose by these 12 cultures might be attributed to the carry-over of glucose in the inoculum. After 10 serial subcultures in GYE medium at $\mathbf{7 2} \mathrm{hr}$. intervals the turbid cultures still failed to produce cellulose pellicles, whereas the parent culture produced submerged zoogloeal material in $24 \mathrm{hr}$., followed by a surface cellulose pellicle in $36-48 \mathrm{hr}$.; the medium below these pellicles remained virtually clear. When the diffusegrowing mutant cultures were plated (GYE agar) colonies were observed which were quite different from those of the parent culture. Parent colonies were punctiform, about $1 \mathrm{~mm}$. in diameter, pale brown in colour, circular, convex, with a regular edge and tough consistency; they appeared on the plates after 3-5 days incubation. In contrast, mutant colonies were 2-4 $\mathrm{mm}$. in diameter, white, circular, raised to convex, with a regular edge and butyrous consistency; they appeared on the plates within $24 \mathrm{hr}$. of inoculation.

An experiment with ethylene glycol in place of ethanol also showed diffuse growth with no visible cellulose pellicle even after 10 subcultures in GYE medium. No growth was observed in media which contained $n$-propanol in place of ethanol.

Shaken cultures in GYE medium. The morphology of the cellulose bodies formed in the shake cultures was similar to that reported by Schramm \& Hestrin (1954); the cellulose appeared as stellate bodies which varied in diameter from 2 to $8 \mathrm{~mm}$. In three experiments which consisted of ten, seven and six serial transfers, respectively, cellulose was produced in all the static and shaken cultures. The lack of agreement between these results and those of Schramm \& Hestrin (1954) may have been because different species of Acetobacter were used.

Nevertheless, the shaken cultures were plated out at each transfer and isolates were picked to tubes of GYE liquid medium in order to examine them for cellulose production. Of 905 isolates tested, the colony forms of which 
appeared identical, only three did not form cellulose pellicles. In these instances the organisms grew diffusely throughout the medium and in $24 \mathrm{hr}$. produced moderate turbidity with no visible cellulose pellicle. In contrast, the cellulose-forming isolates produced submerged zoogloeal material in $24 \mathrm{hr}$., followed by a surface pellicle of cellulose at $36-48 \mathrm{hr}$. When plated, the diffuse cultures gave mutant colonies similar to those described earlier.

Shaken cultures in EYE medium. Two tests gave no visible growth while a third gave diffuse growth but no pellicle formation. When the diffuse culture was plated (GYE medium) the colony type and its growth rate differed from that of the parent culture. Eighty-six colonies were picked and transferred to GYE medium to examine their ability to produce cellulose pellicles. About $70 \%$ of these isolates produced a weak pellicle after 5 days of incubation, and showed a moderate degree of diffuse growth; on transfer to fresh medium they did not form a pellicle but showed diffuse growth. The remaining $30 \%$ continued to produce diffuse growth and did not show pellicle production even after ten serial subcultures.

\section{The origin of celluloseless mutants}

In these experiments the loss of ability to produce a cellulose pellicle might have been induced by the specific treatment (shaking, presence of ethanol) or the cultural conditions may have favoured the growth of mutants already present in the parent culture. Therefore experiments were made to examine for the presence of celluloseless mutants in static GYE cultures. The selective medium used in this work allowed the growth of mutants but not of parent organisms. (The addition of phosphate or magnesium ion to the selective medium allowed growth of the parent culture).

Plating on the selective medium for mutants. Suspensions of organisms (see methods) prepared from three parent GYE cultures, were examined for the presence of mutants. The inoculum consisted of $c .1 \cdot 7( \pm 0 \cdot 3) \times 10^{6}$ bacteria/ plate. The results (Table 1 ) showed that colonies appeared on 14 of the 18 plates prepared. The average number of colonies/plate was 2.06 ; accordingly, the

Table 1. Plating of Acetobacter acetigenum NCIB 8132 on the selective medium

\begin{tabular}{|c|c|c|c|}
\hline \multirow[b]{2}{*}{ Plate no. } & \multicolumn{3}{|c|}{ No. of colonies/plate } \\
\hline & $\begin{array}{l}\text { Suspension* } \\
\text { no. } 1\end{array}$ & $\begin{array}{l}\text { Suspension } \\
\text { no. } 2\end{array}$ & $\begin{array}{c}\text { Suspension } \\
\text { no. } 3\end{array}$ \\
\hline 1 & 1 & 0 & 1 \\
\hline 2 & $\mathbf{3}$ & 9 & 1 \\
\hline 3 & 1 & 12 & 1 \\
\hline 4 & 1 & $\mathbf{2}$ & 2 \\
\hline 5 & $\mathbf{0}$ & 1 & 2 \\
\hline 6 & $\mathbf{0}$ & 1 & $\mathbf{0}$ \\
\hline Total & 6 & 25 & 7 \\
\hline Mean/plate & 1.0 & $4 \cdot 1$ & $1 \cdot 1$ \\
\hline Over-all mean/plate & $2 \cdot 06$ & - & - \\
\hline
\end{tabular}

* Each suspension of organisms was prepared from a culture grown from a single-colony isolate. 
estimated ratio of mutant to parent organisms was 1 mutant to $8 \cdot 2 \times 10^{5}$ parent organisms. The organisms grew rather slowly; the first colonies appeared in about 3 days. All of the $\mathbf{3 8}$ colonies which appeared on the plates were transferred to GYE liquid medium to test for cellulose production, and of these 31 produced a pellicle and 7 showed diffuse growth with no visible cellulose pellicle.

Replica plating. Although some fairly good replicas were obtained on the selective medium, three attempts to obtain a celluloseless mutant by this method were unsuccessful.

Mass plating. The results of these tests are given in Table 2. After $24 \mathrm{hr}$. of incubation c. $50 \%$ of the plates showed the growth of some mutants. The age of the culture tested did not appear to influence the results significantly. The majority of plates showed confluent mutant growth which covered about one-half of the area of the plate; after 3-4 days of incubation parenttype colonies appeared.

Table 2. Results of ' mass-plating' of Acetobacter acetigenum NCIB 8132 on GYE medium

$\begin{array}{cccc}\text { Test no } & \begin{array}{c}\text { Culture } \\ \text { age (days) }\end{array} & \begin{array}{c}\text { No. of } \\ \text { plates }\end{array} & \begin{array}{c}\text { No. of plates } \\ \text { with mutants }\end{array} \\ 1 & 12(0 \cdot 2)^{*} & 12 & 5 \\ 2 & 5(0 \cdot 2) & 12 & 8 \\ 3 & 4(0 \cdot 1) & 7 & 3 \\ 4 & 2(0 \cdot 1) & 6 & 3 \\ & \text { Total } & 37 & 19 \\ & * \text { Volume (ml.) of culture tested. }\end{array}$

Experiments with Acetobacter spp. NCIB 5346 and NCIB 7029

The culture of Acetobacter acetigenum NCIB 5346 was of particular interest since it contained both parent and mutant organisms when received from the National Collection of Industrial Bacteria. The initial GYE culture prepared from the lyophile tube showed diffuse growth at $24 \mathrm{hr}$. and no visible cellulose pellicle. After 6 days of incubation a cellulose pellicle was produced. Subsequently, three serial subcultures were made at 6-day intervals. At this time the culture did not give diffuse growth but a surface pellicle of cellulose was present after $48 \mathrm{hr}$. incubation. When this culture was plated (GYE agar) parent colonies were observed which were quite different from those of the celluloseless mutant.

On several occasions during routine subculture on GYE medium (static incubation) cultures of NCIB 5346 and NCIB 7029 lost their ability to produce cellulose pellicles. For this reason, the stock cultures were kept in malt wort medium thereafter. They appeared to be more stable in this medium, since such cultures always retained the ability to form a pellicle even after prolonged subculture. On other occasions, transfers made from cellulose-producing cultures (GYE medium) to fresh medium showed diffuse growth at first (24 hr.) but later (up to 14 days) showed a cellulose pellicle and a marked diminution in turbidity. In all cases, when the diffuse cultures were plated 
mutant colonies were observed which differed from those of the parent culture. Mutant cultures grown from single-colony isolates picked from the plates did not produce a cellulose pellicle even after repeated subculture for about one year.

The loss of cellulose-forming ability was also observed with cultures of NCIB 5346 and NCIB 7029 when they were inoculated into EYE medium or peptone medium. The frequency of this occurrence was not studied because these cultures showed mutants in GYE medium, as mentioned above.

\section{DISCUSSION}

The frequency of the appearance of these celluloseless mutants has been observed to vary with cultural conditions, species and strain. Loss of ability to produce cellulose was observed with cultures of NCIB 8132 when cultivated under conditions which selected against the growth of the parent organism (EYE liquid medium) but not under conditions which favoured the growth of the parent organism (GYE liquid medium). On the other hand, cultures of two other organisms (NCIB 5346 and NCIB 7029) showed mutants under growth conditions which were favourable for the parent culture.

The mass-plating technique indicated the occurrence of celluloseless mutants in static GYE liquid cultures of NCIB 8132. The fact that the mutant had a faster growth rate than the parent on GYE agar medium allowed its detection 24-48 hr. before parent colonies appeared. However, the physiological relationship between these two different types of organism must be somewhat different in GYE liquid medium since cultures which did not produce cellulose pellicles in that medium were not observed.

The fact that some of the colonies which grew on the selective medium (from which it is inferred that these clones contained mutants) formed cellulose when transferred to GYE liquid medium suggested that these colonies were a mixture of parent and mutant organisms; however, the possibility of reversion cannot be excluded. These results also suggest that the parent has a selective advantage over the mutant in GYE liquid medium. Possibly the parent has a faster growth rate than the mutant in the latter medium, although this was not supported by visual observations of growth.

The demonstration of mutants by the mass-plating technique and the failure to obtain mutants by the method of indirect selection (Lederberg \& Lederberg, 1952) are inconsistent. Preliminary studies on this aspect have revealed that the parent culture (in GYE liquid medium) produces a factor which is inhibitory to the growth of mutants. This would appear to offer an explanation for the failure to enrich the population in the liquid medium with the mutants. This is being studied further.

The authors are grateful for the advice and assistance of Dr A. N. Hall. Also, one of us (R.S.) acknowledges the receipt of an Imperial Chemical Industries Research Fellowship. 


\section{REFERENCES}

Creedy, A. E., JowetT, P. \& Walker, T. K. (1954). Formation of D-cellobiose from starch and from other substrates by an Acetobacter species. Chem. \& Ind. (Rev.), 73, 1297.

LEDERBERg, J. \& LEDERBERG, E. M. (1952). Replica plating and indirect selection of bacterial mutants. J. bact. 63, 399.

Schramm, M. \& Hestrin, S. (1954). Factors affecting production of cellulose at the air-liquid interface of a culture of Acetobacter xylinum. J. gen. Microbiol. 11, 123.

Tosic, J. \& Walker, T. K. (1946). Procedure for the characterisation of the acetic acid bacteria. Chem. \& Ind. (Rev.), 65, 104.

Wright H. B. \& Walker, T. K. (1955). Synthesis of melibiose by an Acetobacter species in a lactate-buffered glucose medium. Chem. \& Ind. (Rev.), 74, 18.

(Received 31 October 1956) 